doi: http://dx.doi.org/10.7124/bc.0009AA

\section{Hi-C analysis of genome folding in individual Drosophila cells}

Sergey V. Ulianov ${ }^{1,2}$, Vlada V. Zakharova $^{2}$, Aleksandra A. Galitsyna ${ }^{2,3,4}$, Kirill E. Polovnikov 2,4 , Ekaterina E. Khrameeva $^{3,4}$, Mariya D. Logacheva ${ }^{2,4}$, Elena A. Mikhaleva ${ }^{5}$, Yegor S. Vassetzky ${ }^{6}$, Aleksey A. Gavrilov ${ }^{1}$, Yuri Y. Shevelev ${ }^{5}$, Sergey K. Nechaev ${ }^{7,8}$, Sergey V. Razin 1,2

${ }^{1}$ Institute of Gene Biology RAS, 119334 Moscow, Russia; ${ }^{2}$ M.V. Lomonosov Moscow State University, 119992 Moscow, Russia; ${ }^{3}$ Institute for Information Transmission Problems (the Kharkevich Institute) RAS, 127051 Moscow, Russia; ${ }^{4}$ Skolkovo Institute of Science and Technology, 143026 Skolkovo, Russia; ${ }^{5}$ Department of Molecular Genetics of Cell, Institute of Molecular Genetics RAS, Moscow, Russia; ${ }^{6}$ Institut Gustave Roussy, CNRS, Villejuif 94805, France; ${ }^{7}$ J.-V. Poncelet Laboratory, CNRS, UMI 2615, 119002 Moscow, Russia; ${ }^{8}$ P.N. Lebedev Physical Institute RAS sergey.v.razin@usa.net

The recent data suggest that the areas of enhancer action are restricted by partitioning of the genome into topologically-associating domains, TADs. However, most of the observations on the $3 \mathrm{D}$ genome organization have been made by conventional methods in cell population where only average characteristics of the so-called typical cell can be identified. On the other hand, FISH-based studies demonstrated that distances between various genomic regions vary significantly in individual cells. To get further insights into the func- tional significance of 3D genome organization it is necessary to estimate the plasticity of this organization. The high throughput chromosome conformation capture protocol (Hi-C) has been modified recently to allow construction of chromatin contact frequency maps for individual cells. Using this modified protocol we have constructed $\mathrm{Hi}-\mathrm{C}$ maps for 20 Drosophila cells (line Dm-BG3c2). In the best cell we have captured $\sim 12 \%$ of the theoretically available contacts. To analyze these sparse contact matrices, we have developed program tools allowing us to take into account the noise by comparing maps from individual cells with artificially generated random matrices. The results of our analysis demonstrate that contact chromatin domains and chromatin compartments do exist in individual Drosopila cells. We also observed the hierarchical organization of contact chromatin domains in individual cells. Consequently this hierarchical organization does not represent a population average but is an intrinsic feature of chromatin folding. The population TAD borders are well reproduced in individual cells and correlate with certain epigenetic signatures. Presence of TADs in individual cells argues that TADs do not represent a population average as postulated by chromatin loop extrusion model but rather originate due to condensation of chromatin domains possessing specific epigenetic signatures.

This work was supported by a Russian Science Foundation grant \#19-14-00016 and RFBR grant 18-29-13013. 